\begin{tabular}{|c|l|}
\hline III Simpósio Paranaense de Modelagem, & Artigo: 26 \\
\cline { 2 - 2 } $\begin{array}{c}\text { Simulaçăo Controle de Processos } \\
\text { ISSN : 1984-7521 }\end{array}$ & Páginas: 190-197 \\
\cline { 2 - 2 }
\end{tabular}

\title{
Análise do Mecanismo Cinético na Modelagem da Combustão de Metano em Microcanais
}

\author{
Evandro A. Garbin ${ }^{1}$, Éliton Fontana ${ }^{1}$ \\ 1- Universidade Federal do Paraná - UFPR - PR
}

\begin{abstract}
Resumo - A combustão é um processo muito importante na engenharia química pois na maioria das vezes é ela a fonte de calor para inúmeros fins. Quando a combustão ocorre em dispositivos com dimensões reduzidas, o controle do processo é fundamental, pois a chama pode facilmente se extinguir devido à alta troca térmica com o ambiente. A utilização de modelos cinéticos que descrevam a reação adequadamente é fundamental, porém a aplicação de modelos muito complexos implica em um aumento significativo no gasto computacional. No presente trabalho foram avaliados dois modelos cinéticos para descrever a combustão de metano em microcanais, um contendo 5 reações e outro contendo 25. Através dos resultados, foi possível observar que o modelo de 5 reações tende a subestimar o calor gerado em comparação com o modelo mais complexo, indicando que par análises onde é necessário conhecer a distribuição de temperatura com detalhes os modelos mais detalhados devem ser aplicados.
\end{abstract}

Palavras chave: microcanais, microcombustor, combustão, cinética de combustão.

\section{Introdução}

O recente desenvolvimento das micro tecnologias, possibilitou o desenvolvimento de vários dispositivos, com funcionamento complexo e de grande utilidade. Mas, muitos destes dispositivos requerem energia para funcionar, a qual pode variar de pequenos miliwatts a centenas de watts, portanto, é necessária uma fonte para prover a energia necessária. Neste campo, entra o desenvolvimento de sistemas micro-termofotovoltáicos (MTPV) para substituir as baterias, que possuem já um mercado bastante rentável, chegando à casa dos $\$ 37$ bilhões por ano [1].

Estes dispositivos utilizam a energia térmica gerada em um micro-combustor como fonte de emissão de fótons para a conversão em energia elétrica. Todo o trabalho no desenvolvimento dos micro combustores é motivado pelo fato de combustíveis de hidrogênio e hidrocarbonetos apresentarem uma densidade de energia muito maior que as mais avançadas baterias de lítio-íon [2].

Quando estudados processos que envolvem a combustão, é indispensável conhecer o comportamento reacional e do sistema, para que possa haver controle e otimização do processo, pois, dependendo das características de cada caso, diferentes fenômenos podem influenciar. Assim, é necessário entrar no mérito da estabilidade da combustão em micro escala.

A micro escala faz com que os problemas associados a combustão venham a ocorrer com maior frequência. As chamas formadas pela combustão de misturas de hidrocarbonetos e ar, usualmente são extinguidas quando frente à espaços com dimensões menores que 1-2 mm [3]. É necessário um controle de estabilidade térmica e química do sistema para que uma combustão estável possa se estabelecer [4].

Quando a taxa de perda de calor para o ambiente é maior que a taxa de geração de calor pela combustão, pode ocorrer a extinção térmica da chama. Além disso, se o material que compõe as paredes do equipamento não for completamente inerte, pode ocorrer a absorção de radicais ativos e a consequente diminuição da taxa de reação. Caso a absorção de radicais for muito intensa, esta diminuição pode induzir a extinção química da chama. Estes fenômenos também ocorrem em combustores convencionais, no entanto devido à baixa área superficial em relação ao volume de reação, podem na maioria dos casos serem desprezados [5].

O presente trabalho, será focado na análise numérica de uma micro combustão de uma mistura $\mathrm{CH}_{4}$-ar e como variações nas condições de contorno e modelagem cinética, influenciam nos resultados da combustão. A utilização de modelos cinéticos detalhados aumenta expressivamente o gasto computacional envolvido, por isso é importante definir 
condições onde modelos simplificados possam ser utilizados e quais as diferenças nas variáveis de interesse quando diferentes modelos são adotados. Os dados obtidos serão comparados à resultados apresentados na literatura [6] para elaboração de futuras conclusões. Um dos motivos da escolha do metano como combustível, é que por se tratar de um combustível muito estudado, possui um banco de dados cinético bastante amplo, possibilitando a análise de diferentes modelos.

\section{Modelagem e formulação numérica}

O domínio de solução avaliado é um microcombustor cilíndrico com as dimensões conforme a imagem abaixo:

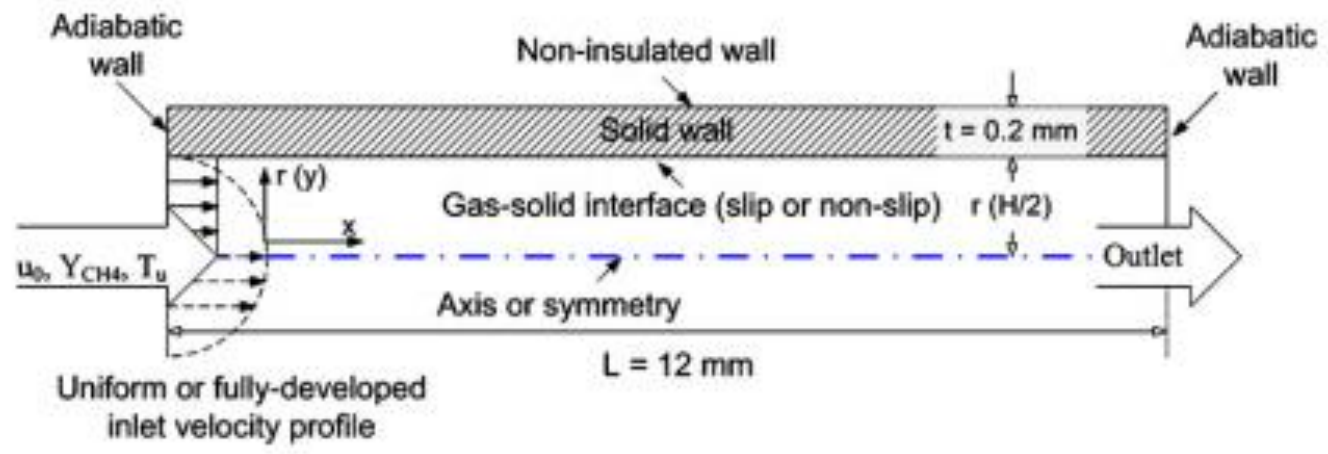

Figura 01: Dimensões e configuração do microcombustor. Fonte: [6]

A partir das equações de conservação de energia, momento, massa e espécie químicas, foram assumidas algumas considerações para desenvolvimento do modelo matemático conforme [6]: (1) Desprezo do efeito Dufour [7]; (2) Sem troca de calor por radiação na fase gás [8;9]; (3) Desconsiderado efeitos causados pela pressão e forças viscosas; (4) Estado estacionário. Assim temos:

- Momento em x:

$\frac{\partial(\rho u u)}{\partial x}+\frac{1}{r} \frac{\partial(\rho u v r)}{\partial r}=-\frac{\partial \rho}{\partial x}+\frac{\partial}{\partial x}\left(\frac{4}{3} \mu \frac{\partial u}{\partial x}\right)+\frac{1}{r} \frac{\partial}{\partial r}\left(r \mu \frac{\partial u}{\partial r}\right)-\frac{\partial}{\partial x}\left(\frac{2 \mu}{3 r} \frac{\partial(v r)}{\partial r}\right)+\frac{1}{r} \frac{\partial}{\partial r}\left(r \mu \frac{\partial v}{\partial x}\right)$

- Momento em r:

$\frac{\partial(\rho u v)}{\partial x}+\frac{1}{r} \frac{\partial(\rho v v r)}{\partial r}=$
$-\frac{\partial \rho}{\partial r}+\frac{\partial}{\partial x}\left(\mu \frac{\partial u}{\partial r}\right)-\frac{1}{r} \frac{\partial}{\partial r}\left(\frac{2 r \mu}{3} \frac{\partial u}{\partial x}\right)+\frac{\partial}{\partial x}\left(\mu \frac{\partial v}{\partial x}\right)+\frac{1}{r} \frac{\partial}{\partial r}\left(\frac{4 r \mu}{3} \frac{\partial v}{\partial r}\right)-\frac{1}{r} \frac{\partial}{\partial r}\left(\frac{2}{3} \mu v\right)$

-Balanço de energia na fase fluida:

$$
\begin{gathered}
\frac{\partial}{\partial x}(\rho u h)+\frac{1}{r} \frac{\partial}{\partial r}(\rho v h r)= \\
\frac{\partial}{\partial x}\left(k \frac{\partial T}{\partial x}\right)+\frac{1}{r} \frac{\partial}{\partial r}\left(k \frac{\partial T}{\partial r} r\right)-\frac{1}{r} \frac{\partial}{\partial r}\left(r \rho \sum_{i=1}^{N} Y_{i} h_{i} V_{i}\right)-\frac{\partial}{\partial x}\left(\rho \sum_{i=1}^{N} Y_{i} h_{i} U_{i}\right)+q
\end{gathered}
$$

- Conservação da espécie química:

$\frac{\partial\left(\rho u Y_{i}\right)}{\partial x}+\frac{1}{r} \frac{\partial\left(\rho v r Y_{i}\right)}{\partial r}=\frac{\partial}{\partial x}\left[D i \frac{\partial\left(\rho Y_{i}\right)}{\partial x}\right]+\frac{1}{r} \frac{\partial}{\partial r}\left[\operatorname{Dir} \frac{\partial\left(\rho Y_{i}\right)}{\partial r}\right]+\omega i$ 
- Balanço de energia na fase sólida:

$\frac{\partial}{\partial x}\left(k_{s} \frac{\partial T_{s}}{\partial x}\right)+\frac{1}{r} \frac{\partial}{\partial r}\left(k_{s} \frac{\partial T_{s}}{\partial r} r\right)=0$

- Continuidade:

$\frac{\partial(\rho u)}{\partial x}+\frac{1}{r} \frac{\partial(\rho v r)}{\partial r}=0$

Onde nas equações o significado de cada incógnita é: $\rho$ densidade da mistura, $u$ velocidade no eixo $\mathrm{x}, \mathrm{r}$ raio do microcombustor, $\mathrm{v}$ velocidade no eixo $\mathrm{y}, \mu$ viscosidade dinâmica, $h$ entalpia, $k$ condutividade térmica do gás, $T$ temperatura, $Y_{i}$ fração molar de cada espécie, $h_{i}$ entalpia de cada espécie, $U_{i}$ velocidade no eixo $\mathrm{x}$ de cada espécie, $V_{i}$ velocidade no eixo y de cada espécie, q taxa de geração de calor por unidade de volume, Di coeficiente de difusão de cada espécie, $\omega_{\mathrm{i}}$ taxa de produção de cada espécie, $\mathrm{k}_{\mathrm{s}}$ condutividade térmica do sólido, $\mathrm{T}_{\mathrm{s}}$ temperatura do sólido.

Dois mecanismos cinéticos foram escolhidos para as simulações. O primeiro contém 7 espécies envolvidas em 5 reações que resultam na oxidação do metano, sendo apresentado na Tabela 01. As respectivas reações juntamente com seus dados e constantes (para a equação de Arrhenius) estão apresentados abaixo e foram obtidos em [10]. O segundo mecanismo envolve 16 espécies químicas e 25 reações reversíveis, podendo ser encontrado em Li et al. [6]. A utilização do segundo mecanismo aumenta o tempo computacional em aproximadamente 7 vezes.

Tabela 01: Mecanismo cinético com 5 reações.

\begin{tabular}{|c|c|c|c|c|}
\hline \multicolumn{2}{|c|}{ Reações } & E. de Ativação $(\mathrm{m}, \mathrm{kmol}, \mathrm{s})$ & Exp. de Temperatura & $\begin{array}{c}\text { Fator Pre-exp. } \\
\text { da Reação } \\
(\mathrm{J} / \mathrm{kmol})\end{array}$ \\
\hline 1 & $\begin{array}{c}\mathrm{CH} 4+3 / 2 \mathrm{O} 2 \rightarrow \\
\mathrm{CO}+2 \mathrm{H} 2 \mathrm{O}\end{array}$ & $1,72 \mathrm{E}+08$ & 0 & $1,6596 \mathrm{E}+15$ \\
\hline 2 & $\mathrm{CO}+1 / 2 \mathrm{O} 2 \rightarrow \mathrm{CO} 2$ & $9,654 \mathrm{E}+07$ & 0 & $9,9799 \mathrm{E}+14$ \\
\hline 3 & $\mathrm{CO} 2 \rightarrow \mathrm{CO}+1 / 2 \mathrm{O} 2$ & $5,1774 \mathrm{E}+08$ & 0 & $2,2336 \mathrm{E}+14$ \\
\hline 4 & $\begin{array}{c}\mathrm{N} 2+\mathrm{O} 2+\mathrm{CO} \rightarrow \\
2 \mathrm{NO}+\mathrm{CO}\end{array}$ & $4,4366 \mathrm{E}+08$ & 0 & $8,8308 \mathrm{E}+23$ \\
\hline 5 & $\mathrm{~N} 2+\mathrm{O} 2 \rightarrow 2 \mathrm{NO}$ & $5,7276 \mathrm{E}+08$ & $-0,5$ & $9,2683 \mathrm{E}+14$ \\
\hline
\end{tabular}

As equações juntamente com o mecanismo reacional, foram discretizadas utilizando o método dos volumes finitos e resolvidos pelo software computacional Fluent ${ }^{\circledR}$ Release 18.1. As equações foram resolvidas em uma configuração $2 \mathrm{D}$ com eixo axial. $\mathrm{O}$ software resolve primeiro a equação de momento, então a de continuidade para então considerar os fluxos de massa e pressão. Por fim, o balanço de energia e a equação de conservação química são solucionados a partir de iterações que podem ser monitoradas até que o resultado convirja. O critério de convergência utilizado foi de $10^{-3}$ para continuidade, $10^{-6}$ para a velocidade e $10^{-3}$ para a concentração das espécies. Foi considerada lei de gases ideais para calculo da densidade dos gases. A viscosidade, calor específico e condutividade térmica do gás foi calculado por um modelo de média ponderada em massa ponderada de todas as espécies, porém o calor especifico de cada espécie foi calculado utilizando um ajuste polinomial dependente da temperatura.

As paredes do cilindro foram definidas como de aço e com condutividade térmica de $20 \mathrm{~W} /\left(\mathrm{m}^{2} \mathrm{~K}\right)$, com uma espessura de $0.2 \mathrm{~mm}$ e comprimento de $12 \mathrm{~mm}$. Na entrada, a 
mistura $\mathrm{CH}_{4}$-ar entra à $300 \mathrm{~K}$. As paredes à esquerda e direita foram definidas como adiabáticas e a perda de calor ocorre pela parede externa. Os coeficientes de troca convectiva e emissividade são respectivamente $5 \mathrm{~W} /\left(\mathrm{m}^{2} \mathrm{~K}\right)$ e 0,2 . Para inicializar o problema, foi necessário colocar uma alta temperatura, para garantir que a chama não se extinguisse, foi colocada uma temperatura de $1500 \mathrm{~K}$.

Foi variado também o tamanho do microcombustor e os resultados respectivos serão na sequencia apresentados. A fração de alimentação foi definida conforme [6] em mol de 0,05 para o metano e 0,18 para o oxigênio.

\section{Resultados e discussões}

Para avaliar a influência do modelo cinético nas características gerais do processo de combustão, determinou-se os perfis de diversas variáveis de interesse ao longo do canal. Inicialmente, considerou-se o combusto com $1 \mathrm{~mm}$ de diâmetro. Na Fig 02 são apresentados os perfis de fração mássica de metano para uma velocidade de alimentação de $0.5 \mathrm{~m} / \mathrm{s}$, considerando o modelo cinético com 5 reações (Fig 02-a), o modelo com 25 reações (Fig 02-b) e os resultados apresentados por Li et al. [6], reproduzidos aqui para comparação.



(a) Modelo cinético 1 (5 reações)

(b) Modelo cinético 2 (25 reações)

(c) Li et al. [6]

Figura 02: Perfis de fração mássica de metano para os diferentes modelos cinéticos avaliados, considerando $u=0.5 \mathrm{~m} / \mathrm{s}$.

Como pode ser observado, o modelo cinético com 5 reações prevê um consumo mais rápido do combustível, porém, os três casos mostram que todo o metano é consumido logo no início do reator. Considerando que Li et al. [6] utiliza o mesmo modelo cinético de 25 reações utilizado na Fig 02-b, existe uma diferença entre os perfis obtidos, o que provavelmente está relacionado com o tratamento dado ao escoamento. Os perfis apresentados por $\mathrm{Li}$ et al [6] foram obtidos considerando um escoamento laminar, enquanto que para a Fig 02-b considerou-se o modelo $k-\varepsilon$ para modelar a turbulência. Apesar de o número de Reynolds pode chegar a, no máximo, aproximadamente 2.000 , a utilização de modelos de turbulência é comum para captar a influência do grau de mistura na reação. Na Figura 03 são apresentados os perfis de velocidade obtidos neste trabalho e o valor apresentado por $\mathrm{Li}$ et al. [6].

Os perfis de velocidade obtidos são bastante similares, em particular entre o modelo com 25 reações (Fig 03-b) e o apresentado por Li et al. [6]. Para o modelo de 5 reações, a velocidade máxima obtida é levemente inferior, mostrando que a cinética possui um papel significativo na determinação do campo de velocidades. 
$\mathrm{Na}$ Figura 04 são apresentados os perfis de temperatura para os dois modelos cinéticos. Neste caso, ocorre uma maior diferença entre os valores obtidos considerando 5 e 25 reações, sendo que o modelo de 25 reações prevê uma temperatura média superior. Como o aumento na temperatura está associado com o calor de reação, é normal que esta seja muito dependente do modelo utilizado. Porém, observa-se que mesmo em comparação com o resultado apresentado por $\mathrm{Li}$ et al. [6], que também utiliza o modelo com 25 reações, a temperatura na Fig 04-b é em média maior. Este comportamento está associado com o maior grau de mistura prescrito pelo modelo $k-\varepsilon$, o que faz com que a distribuição de energia seja mais homogênea.

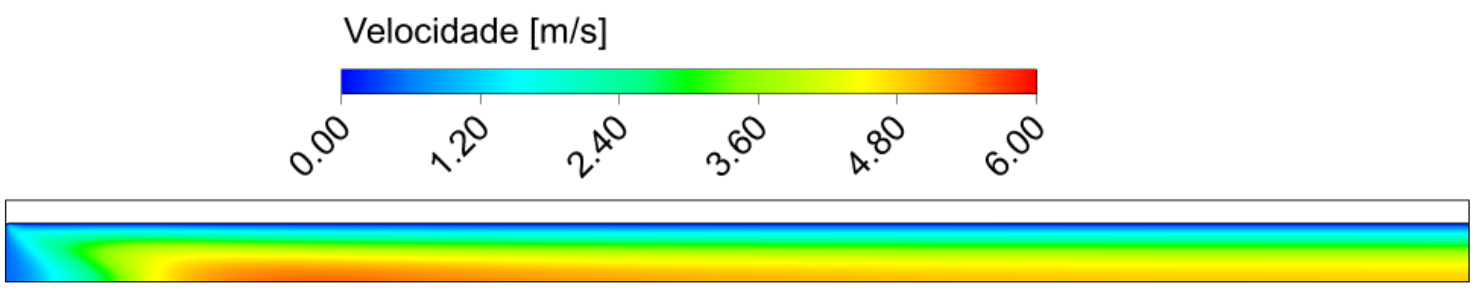

(a) Modelo cinético 1 (5 reações)

(b) Modelo cinético 2 (25 reações)

(c) Li et al. [6]

Figura 03: Perfis de velocidade para os diferentes modelos cinéticos avaliados, considerando $u=0.5 \mathrm{~m} / \mathrm{s}$.

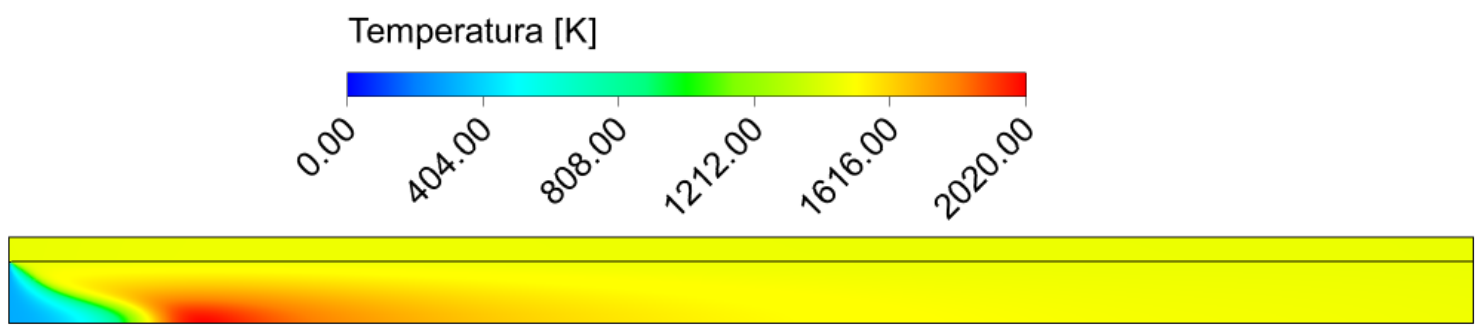

(a) Modelo cinético 1 (5 reações)

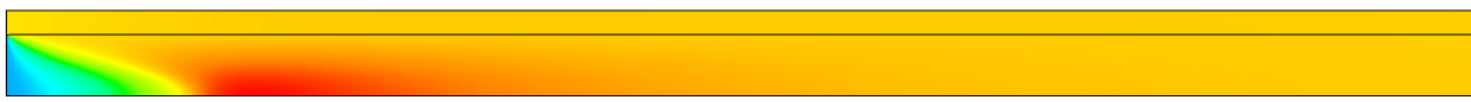

(b) Modelo cinético 2 (25 reações)



(c) Li et al. [6]

Figura 04: Perfis de temperatura para os diferentes modelos cinéticos avaliados.

Para a aplicação em dispositivos termo-fotovoltáicos, é importante que a temperatura da parede externa seja a mais alta possível, portanto o principal ponto na modelagem do processo é conseguir prever adequadamente esta temperatura. Na Figura 05 são apresentadas as curvas de temperatura ao longo da direção x para casos envolvendo um combustor com diâmetro de $1 \mathrm{~mm}$ com velocidade de alimentação de $0.5 \mathrm{~m} / \mathrm{s}$ e um 
combustor com diâmetro de $2 \mathrm{~mm}$ e velocidade de alimentação de $1 \mathrm{~m} / \mathrm{s}$. A dependência da temperatura da parede externa com o diâmetro segue a mesma tendência para os dois modelos cinéticos, sendo que para o combustor com maior diâmetro a temperatura é razoavelmente maior devido ao aumento na vazão de alimentação de combustível. Em ambos os casos, o modelo com 25 reações previu uma temperatura aproximadamente 200 $\mathrm{K}$ superior, o que representa um aumento de mais de quase $15 \%$.

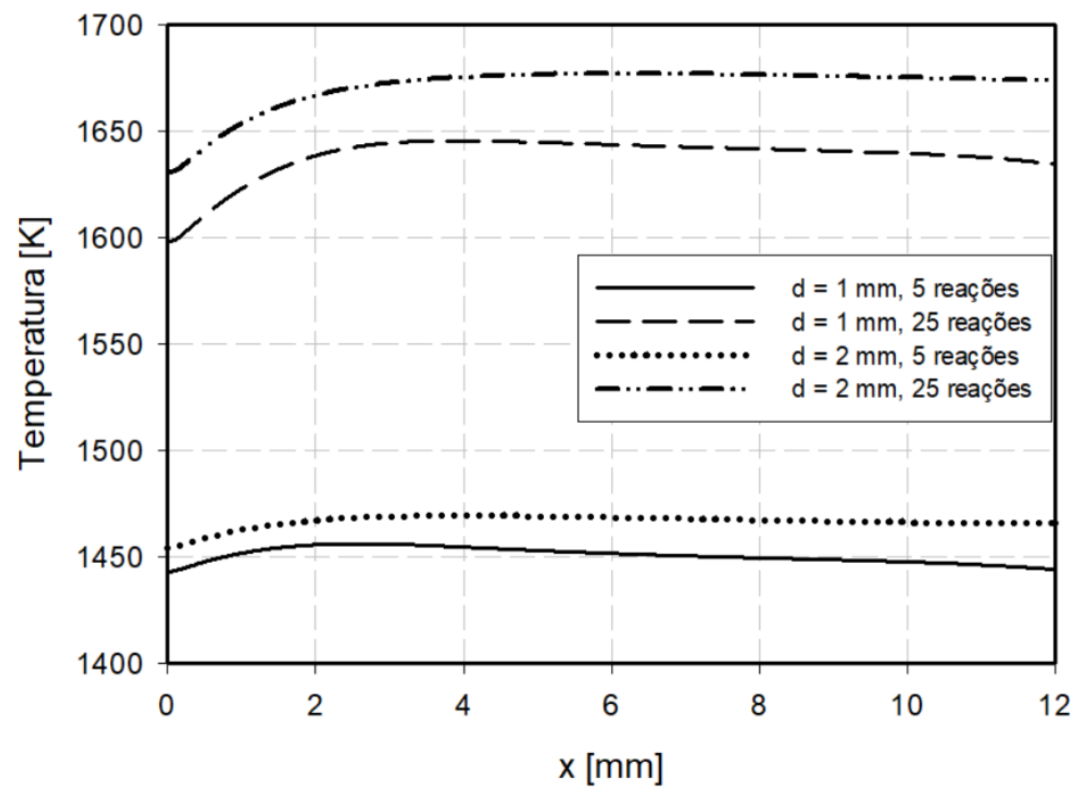

Figura 05: Perfis de temperatura na parede externa do combustor.

Apesar do desvio observado na temperatura da parede externa, quando avaliada a temperatura na linha central do sistema os modelos cinéticos apresentam resultados mais próximos, como mostrado na Figura 06. Para o combustor com diâmetro de $1 \mathrm{~mm}$, os desvio ocorrem principalmente após o ponto de máximo da temperatura, sendo que próximo à saída a diferença é de aproximadamente $200 \mathrm{~K}$, justificando a diferença observada na Figura 05. Já para o combustor com diâmetro de $2 \mathrm{~mm}$ ocorrem diferenças mais próximas à entrada, indicando que o modelo com 25 reações prevê que as reações iniciam mais rapidamente. Porém, o ponto de máxima temperatura coincide para os dois modelos e após este ponto o comportamento é semelhante para os dois casos.

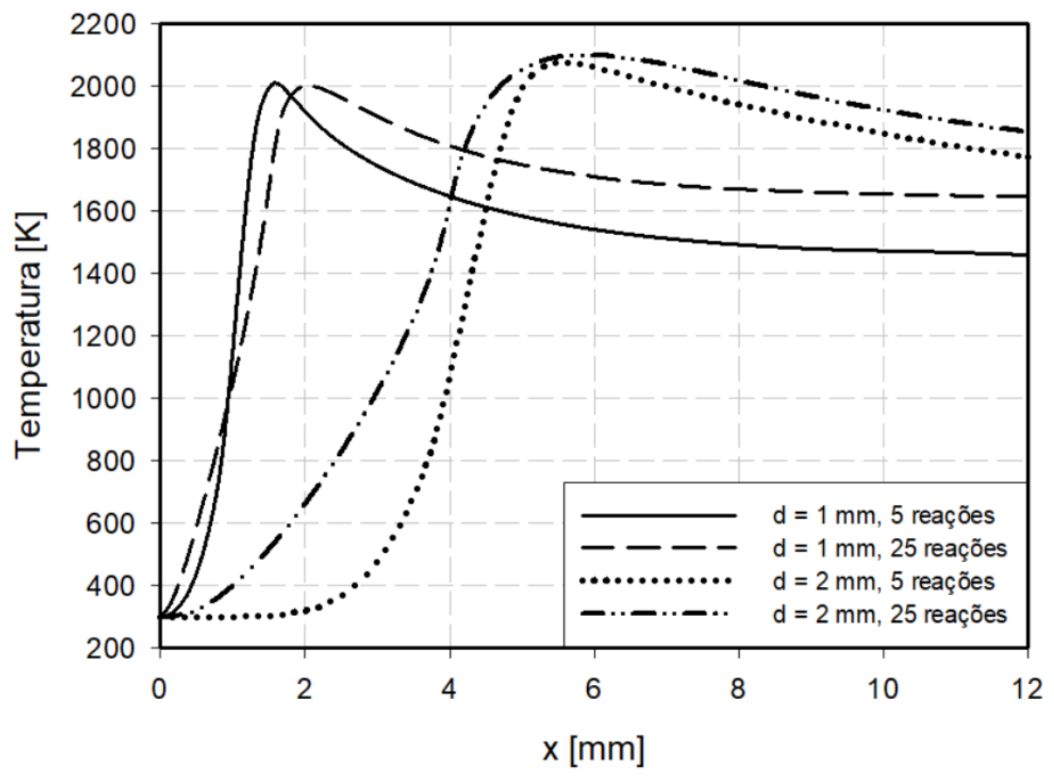

Figura 06: Perfis de temperatura ao longo do eixo central do combustor. 


\section{Conclusão}

Neste trabalho avaliou-se a combustão de metano em microcanais através do uso de dois modelos cinéticos distintos, sendo um deles um modelo simplificando contendo 7 espécies químicas e 5 reações e outro um modelo mais complexo com 16 espécies químicas e 25 reações, sendo várias delas reversíveis. O sistema de equações governantes foi resolvido com o uso do software ANSYS Fluent 18.1.

A análise dos resultados mostra que mesmo o modelo mais simples consegue prever aspectos importantes do sistema, como o consumo total de combustível e a localização dos pontos de máxima temperatura, além de gerar um comportamento qualitativo condizente com os modelos mais complexos. No entanto, para estimar com precisão os valores de temperatura em regiões específicas, os modelos simplificados geram desvio significativos. Para o caso da temperatura da parede externa, obteve-se um valor aproximadamente $15 \%$ superior quando o modelo mais complexo foi utilizado. Para a aplicação em sistemas termo-fotovoltáicos, onde esta temperatura ser determinada com detalhes, este desvio é muito significativo, portanto deve-se optar pela utilização de modelos mais complexos para que o processo possa ser descrito com o nível de detalhamento necessário.

\section{Referências Bibliográficas}

[1] Dunn-Rankin D, Leal EM, Walther DC. Personal power systems. Progress in Energy and Combustion Science 2005; 31(5e6):422e65

[2] P.D. Ronney. Analysis of non-adiabatic heat-recirculating combustors, Combustion and Flame 135 (2003) 421-439.

[3] NORTON, D. G.; VLACHOS, D. G. A CFD study of propane/air microflame stability. Combustion and Flame, v. 138, p. 97 - 107, 2004.

[4] MARUTA, K. Micro and mesoscale combustion. Proceedings of the Combustion Institute, v. 33, p. 125 - 150, 2011.

[5] HUA, J.; WU, M.; SHAN, X. Studies on combustion characteristics in microcombustor and its applications. [S.1.]: Nova Science Publishers, 2007. (Proceedings of the Combustion Institute).

[6] LI, J.; CHOU, S K.; YANG, W.M.; LI, Z. W., A numerical study on premixed microcombustion of $\mathrm{CH} 4$-air mixture: Effects of combustor size, geometry and boundary conditions on flame temperature, Chemical Engineering Journal, v. 150, p. 213 - 222, 2009.

[7] F.A. Williams, Combustion Theory: Fundamental Theory of Chemical Reacting Flow Systems, Benjamin/Cummings, Menlo Park, 1985

[8] D.G. Norton, D.G. Vlachos, A CFD study of propane/air microflame stability, Combust. Flame 138 (2004) 97-107. 
[9] D.G. Norton, D.G. Vlachos, Combustion characteristics and flame stability at the microscale: a CFD study of premixed methane/air mixtures, Chem. Eng. Sci. 58 (2003) $4871-4882$.

[10] User Guide for ANSYS Fluent Release 18.1, ANSYS Inc Ltd. 